\section{E-214 ENDOVASCULAR TREATMENT OF DISSECTING VERTEBRAL ARTERY ANEURYSMS: A 20-YEAR INSTITUTIONAL EXPERIENCE}

J Catapano*, C Nguyen, C Korson, J Baranoski, T Cole, N Majmundar, D Wilkinson, V Fredrickson, F Albuquerque, A Ducruet. Neurosurgery, BNI, Phoenix, AZ

\subsection{6/neurintsurg-2020-SNIS.245}

Background Vertebral artery dissecting aneurysms (VADAs) are a rare cause of subarachnoid hemorrhage associated with high rates of morbidity and mortality. Ruptured non-dominant VADAs are traditionally treated via endovascular coil-occlusion. However, controversy exist for the appropriate management of unruptured VADAs and ruptured dominant VADAs. To the authors knowledge, this is the largest single-center study comparing modern neuroendovascular neurosurgical treatment strategies, including flow diversion (FD), to treat VADAs.

Methods All patients with a VADA treated endovascularly at a single center from January 1st, 1999 to December 31st, 2019 were retrospectively analyzed from a prospectively collected database. VADAs were categorized as either dominant or nondominant vertebral artery. Furthermore, location of the VADA was classified as either the proximal V4 segment of the vertebral artery (proximal to PICA), incorporating PICA origin, or (distal V4) distal to PICA. Primary neurological outcomes were measured via mRS, with a mRS $>2$ categorized as a poor neurological outcome and a decline in mRS from the preoperative neurological exam as a worse neurological outcome. Secondary outcomes included retreatment rate and complications.

Results 91 patients underwent endovascular treatment for a VADA over this 20-year period (44 patients underwent open microsurgical intervention). 77 (85\%) VADAs were on the proxmial V4 segment, 8 (9\%) included the PICA origin, and $6(7 \%)$ arose distal to PICA. Coil-occlusion was performed in 47 (51\%), FD in 29 (32\%), and stent/coil in 15 (17\%) cases. 54 patients $(59 \%)$ presented with SAH (treated via coil-occlusion in $39, \mathrm{FD}$ in 7 , and stent/coil in 8 cases; $\mathrm{p}<0.001$ ) and 44 VADAs (48\%) involved a dominant vertebral artery (all dominant vertebral arteries were treated by either a FD or stent/coil; $\mathrm{p}<0.001)$. Rates of complications and retreatment were both significantly higher in patients treated with stent/ coil ( complication: $\mathrm{N}=4,27 \%$, retreatment: $\mathrm{N}=6,40 \%$ ) vs either coil-occlusion (complication: $\mathrm{N}=1,2 \%$, retreatment $=2$, $4 \%$ ) or FD (complication: $\mathrm{N}=2,7 \%$, retreatment: $\mathrm{N}=4$, $14 \%)(p=0.008$ and $p=0.002$, respectively). Preoperative mRS was significantly higher in patients treated with coil-occlusion (3.2 \pm 1.4$)$ than FDD $(1.9 \pm 1.5)$ or stent/coil $(1.8 \pm 1.3)$ $(\mathrm{p}<0.001)$. Likewise, coil-occlusion $(22,46 \%)$ was associated with a higher percentage of patients with a mRS $>2$ on follow-up than FDD $(4,14 \%)$ or stent/coil $(3,20 \%)(p=0.006)$. For dominant vertebral arteries, stent/coil $(6,40 \%)$ required greater percentage of retreatments than FD (4, 14\%) $(\mathrm{p}=0.049)$. Of the unruptured VADAs $(\mathrm{N}=37), 1$ patient suffered a complication (3\%), 4 patients $(11 \%)$ required retreatment, 2 patients $(5 \%)$ had $\mathrm{mRS}>2$, and 8 patients $(22 \%)$ exhibited a decline in mRS on follow-up, with no significant difference between the treatments.

Conclusion The majority of ruptured VADAs at our center were treated by coil-occlusion of non-dominant vertebral artery. For dominant vertebral arteries, FD required less retreatment than stent/coil cases. Furthermore, endovascular treatment of unruptured VADAs is safe and associated with favorable angiographic and neurological outcomes.
Disclosures J. Catapano: None. C. Nguyen: None. C. Korson: None. J. Baranoski: None. T. Cole: None. N. Majmundar: None. D. Wilkinson: None. V. Fredrickson: None. F. Albuquerque: None. A. Ducruet: None.

\section{E-215 ROTATIONAL ANGIOGRAPHY COMPLICATED BY ANEURYSM RERUPTURE: A CASE SERIES}

'D Nistal*, 'D Wei, ${ }^{2} \mathrm{~J}$ Mascitelli, ${ }^{1} \mathrm{H}$ Shoirah, ${ }^{3} \mathrm{R}$ Starke, ${ }^{4} \mathrm{E}$ Levy, ${ }^{5} \mathrm{~J}$ Howington, ${ }^{1} \mathrm{~J}$ Mocco, ${ }^{1} \mathrm{~T}$ Oxley. "Neurosurgery, Icahn School of Medicine, New York, NY; ${ }^{2}$ Neurosurgery, UT Health San Antonio, San Antonio, TX; ${ }^{3}$ Neurosurgery, University of Miami Health System, Miami, FL; ${ }^{4}$ Neurosurgery, University at Buffalo, Williamsville, NY; ${ }^{5}$ Neurosurgery, Neurological and Spine Institute, Savannah, GA

\subsection{6/neurintsurg-2020-SNIS.246}

Introduction Digital subtraction angiography (DSA) is considered the gold standard for diagnosing intracranial aneurysms. In cases of aneurysmal subarachnoid hemorrhage (aSAH), 3DRA has become standard of care to detect aneurysms and to plan treatment in cases with inconclusive noninvasive imaging. Rerupture during angiography with contrast extravasation is a rare but devastating complication and has a mortality of $50 \%$ to $80 \%$. In this case series, we report our experience of aneurysm rerupture as a complication of 3DRA for aSAH.

Methods The electronic medical records of eight patients across four separate institutions who underwent 3DRA for evaluation of aneurysm after aSAH were reviewed. Data from patient medical charts and their angiographic procedures were reviewed to assess both clinical and angiographic outcomes. Overall case descriptions and patients' histories were reviewed and described in detail.

Results Of the eight cases reviewed, the mean aneurysm size was $9.7 \pm 5.5 \mathrm{~mm}$ and the mean Hunt Hess and modified Fisher Score on arrival were $3.5 \pm 0.96$ and $3.25 \pm 0.83$ respectively. Injection rate, injection volume, and pressure were 3.6 $\pm 1.4 \mathrm{~mL} / \mathrm{s}, 21 \pm 2.1 \mathrm{~mL}$, and $488 \pm 124$ psi. Inpatient mortality after aneurysmal rerupture was $37.5 \%(\mathrm{n}=3)$, with $37.5 \%$ $(n=3)$ requiring EVD placement and 50\% $(n=4)$ requiring craniectomy.

Conclusion This is the first series to report aneurysm rerupture as a complication of 3DRA. Rerupture during 3DRA is a devastating complication that commonly led to patient mortality in our series. Future studies will be needed to further elucidate characteristics of patients associated with 3DRA complications and to compare 3DRA complication rates with other aSAH diagnostic modalities.

Disclosures D. Nistal: None. D. Wei: None. J. Mascitelli: None. H. Shoirah: None. R. Starke: None. E. Levy: None. J. Howington: None. J. Mocco: None. T. Oxley: None.

\section{E-216 THE EFFECT OF OMEPRAZOLE ON PATIENTS TAKING CLOPIDOGREL AFTER FLOW DIVERTER DEVICE PLACEMENT}

J Catapano*, V Fredrickson, A Wakim, J Lundberg, B Hendricks, J Baranoski, T Cole, D Wilkinson, N Majmundar, F Albuquerque, A Ducruet. Neurosurgery, BNI, Phoenix, AZ

10.1136/neurintsurg-2020-SNIS.247

Background Omeprazole is a common proton pump inhibitor (PPI) that interferes with clopidogrel hepatic activation, potentially reducing its platelet inhibition efficacy. In the cardiovascular literature, omeprazole has been reported to increase P2Y12 
reactivity levels and may lead to adverse cardiovascular outcomes in patients treated with drug eluting stents following cardiac catheterization. However, literature on omeprazole taken in association with clopidogrel is lacking in the neuroendovascular literature. We hypothesized that patients taking omeprazole would exhibit higher PRU levels and increased complications following treatment with a flow diverter device (FDD).

Methods All patients with a FDD placement for an intracranial aneurysm at a large tertiary institution from January 1st, 2015 to December 31st, 2018 were retrospectively analyzed. Inclusion criteria included 1) documented clopidogrel administration, 2) available P2Y12 levels, and 3) thorough documentation of administration of other medications including omeprazole. Outcomes analyzed included ischemic stroke on MRI and FDD stenosis on follow-up angiography.

Results Out of a total of of 138 patients that met the inclusion criteria, 16 (12\%) were taking both omeprazole and clopidogrel. The average age for the omeprazole patients was significantly higher than those not taking omeprazole[69( \pm 10$)$ vs 57 $( \pm 14) \quad(\mathrm{p}=0.001)]$. A significantly higher P2Y12 reactivity (decreased platelet inhibition) was observed in patients taking omeprazole $(\mathrm{PRU}=250)$ versus those not taking omeprazole $(P R U=110) \quad(p<0.001)$. Furthermore, a higher number of patients were found to have a P2Y12 level $>180$ PRU in the omeprazole $(\mathrm{N}=14,88 \%)$ vs no omeprazole $(\mathrm{N}=24,20 \%)$ patients $(\mathrm{p}<0.001$, OR 29; 95\% CI 6-134). There were no significant differences in the rates of ischemic strokes, FDD stenosis, or hemorrhagic complications between the two groups. Conclusion Omeprazole significantly increases the P2Y12 reactivity levels in intracranial aneurysm patients on clopidogrel treated with a FDD. However, omeprazole did not increase the risk of ischemic events and/or device stenosis. Nonetheless, given the significant association between omeprazole and decreased clopidogrel efficacy, omeprazole should not be administered to neuroendovascular patients treated with a FDD taking clopidogrel.

Disclosures J. Catapano: None. V. Fredrickson: None. A. Wakim: None. J. Lundberg: None. B. Hendricks: None. J. Baranoski: None. T. Cole: None. D. Wilkinson: None. N. Majmundar: None. F. Albuquerque: None. A. Ducruet: None.

\section{E-217 VOXEL BASED CALCULATION OF ANEURYSM VOLUME AND MORPHOLOGICAL CHARACTERISTICS}

C Settanni* ${ }^{*}$ T Becker, W Merrit. Northern Arizona University, Flagstaff, AZ

10.1136/neurintsurg-2020-SNIS.248

Introduction Various embolization techniques are available for intracranial aneurysms. The volume of an aneurysm is of interest for the device selection and delivery of a variety of embolization techniques, such as: coils, liquid embolics, flow disruptors, and flow diverters. Accurate aneurysm sizing and volumetric information can help interventionalists assess flow and stability pre-treatment, and assess potential remnant or recanalization risks post-treatment.

Materials and Methods This research project applies voxelbased volume calculations, from patient MRI medical imaging data, to determine accurate 3-D aneurysm volume calculations. Additionally, the application can display clinically relevant parameters, such as aneurysm neck diameter, dome height and midline-dome width (for dome: neck (D:N) ratio calculations. To develop the calculations, formalin-fixed canine aneurysms model samples are measured with a Bruker $7 \mathrm{~T}^{\circledR}$ MRI and reconstructed in 3-D. (figure 1).

The images is be processed using a MATLAB ${ }^{\circledR}$ algorithm. This algorithm patches together image segments from MRI and micro CT scans of animals and/or humans. The application acquires 3D MRI data, discretizes the domain intrasaccular aneurysm space, and evaluates the volume of the aneurysm sac. This data is compared to physical measurements of excised aneurysms and calibrated 2-D angiographic images.

Results Imaging processing techniques may be used to determine the domain of aneurysms with unprecedented precision, with less than $5 \%$ volumetric error, whereas current measurement techniques, especially in 2-D planes have errors as high as $30 \%$. This technique helps determine the macroscopic properties of aneurysms, as well as accurately calculate the volumes of heterogeneous features such as blebs or abnormal aneurysm shapes.

Conclusion This project brings together clinical and engineering expertise to translate medical imaging data directly into volumetric measurements with highly precise calculations that are currently not available from 2-D angiographic images. Measuring the size and dimensional properties of aneurysms with voxel-based volume calculations provides a fast, reliable and repeatable resource for aneurysm assessment. This technique will help interventionalists appropriately asses risk and treatment options for a broad variety of aneurysm morphologies.

Disclosures C. Settanni: None. T. Becker: 1; C; STTR (NIH). 2; C; Aneuvas. W. Merrit: 1; C; STTR(NIH). 2; C; Aneuvas.

\section{E-218 STENT-ASSISTED COILING OF ACUTE RUPTURED CEREBRAL ANEURYSMS}

V Berestov*, K Orlov, N Strelnikov, M Demyanovskaya. Neurosurgery department, National Medical Research Center (Meshalkin Clinic), Novosibirsk, Russian Federation

10.1136/neurintsurg-2020-SNIS.249
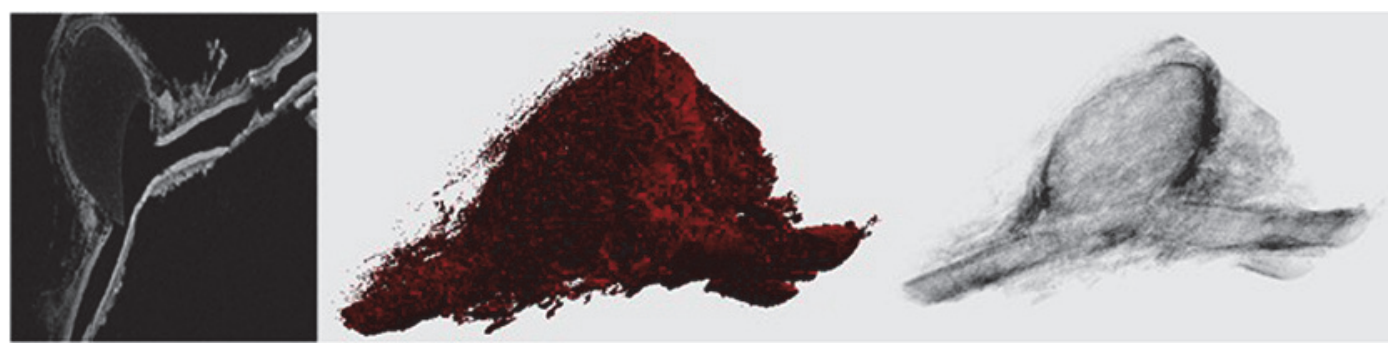

Abstract E-217 Figure 1 Left: 2D MRI longitudinal section of a surgically anastomosed canine sidewall aneurysm (1 month after creation), Middle: Decomposition of the 3D MRI by grouping values of equal brightness, Right: 3D reconstruction of the aneurysm sac 